\title{
Asymmetrical Electroencephalographic Change of Human Brain During Sleep Onset Period
}

\author{
Doo-Heum Park ${ }^{1}$ and Chul-Jin Shin ${ }^{2} \bowtie$ \\ ${ }^{1}$ Department of Psychiatry, Konkuk University School of Medicine, Seoul, Republic of Korea \\ ${ }^{2}$ Department of Neuropsychiatry, College of Medicine and Medical Research Institute, Chungbuk National University, Cheongju, Republic of Korea
}

Objective Human cerebral hemisphere is known to function asymmetrically with daytime left hemisphere superiority in most righthanded persons. It may have relevance to the localization of specific function of the brain. This study attempted to reveal whether the functional cerebral asymmetry in the wakeful state is still maintained throughout the sleep onset period.

Methods Thirty-channel EEG was recorded in 61 healthy subjects. The EEG power spectra of each of the seven frequencies were compared between the two kinds of 30-second states; the wakeful stage and the late-sleep stage 1.

Results The asymmetrical indices of sleep stage 1 at several fronto-central leads were decreased in the delta, theta, alpha-2, and all beta bands. Conversely, at parts of parieto-occipital leads showed an increase in the indices of the theta, alphas, beta-1, and beta-2 bands . Any fronto-central leads did not show an increase in the index, and no parieto-occipital leads showed a decrease.

Conclusion During the sleep onset period, power spectral asymmetry of the brain showed a different pattern from the wakeful stage. This asymmetrical pattern of EEG powers may suggest a reversal of the left hemispheric dominance during sleep.

Psychiatry Investig 2017;14(6):839-843

Key Words Cerebral asymmetry, Sleep onset period, EEG spectra.

\section{INTRODUCTION}

The human brain consists of two hemispheres, and they have been known not to be completely symmetrical with respect to their structural or functional aspects. Structural asymmetry of the brain was reported in the greater left planum temporale, ${ }^{1,2}$ longer left Sylvian fissure, ${ }^{2}$ and greater folding of left frontal operculum, ${ }^{3}$ as well as the superior parietal lobules and angular gyri. ${ }^{4}$ These left-right asymmetries are seen not only in humans, but also in the other greater apes and even in rodents. ${ }^{5}$

The hemispheric asymmetry was also found in their function, in addition to their structure. In an electrophysiological

Received: February 3, 2017 Revised: March 16, 2017

Accepted: May 25, 2017 Available online: October 13, 2017

$\bowtie$ Correspondence: Chul-Jin Shin, MD, PhD

Department of Neuropsychiatry, College of Medicine and Medical Research Institute, Chungbuk National University, 1 Chungdae-ro, Seowon-gu, Cheongju 28644, Republic of Korea

Tel: +82-43-269-6235, Fax: +82-43-267-7951, E-mail: cjshin@chungbuk.ac.kr (a) This is an Open Access article distributed under the terms of the Creative Commons Attribution Non-Commercial License (http://creativecommons.org/licenses/by$\mathrm{nc} / 4.0$ ) which permits unrestricted non-commercial use, distribution, and reproduction in any medium, provided the original work is properly cited. study, Benca et al. ${ }^{6}$ reported frontal alpha asymmetry, and a functional brain imaging study by Swanson et al. ${ }^{7}$ also described functional asymmetry of the default mode network.

During wakefulness, an electroencephalographic (EEG) study observed a greater left frontal absolute power and a greater right occipital delta power compared with the other side. ${ }^{8}$ The other study with fifty-nine healthy subjects reported right side dominance of alpha oscillation on the frontocentral area and vice versa on the parieto-occipital area. ${ }^{9}$ The majority of reported investigations regarding resting EEG asymmetry is about frontal alpha asymmetry, and these studies have suggested that it is the correlates of emotional regulation and flexibility, ${ }^{10,11}$ or stress response and cortisol levels. ${ }^{12-15}$

Regarding the sleep state, a unilateral bright-light exposure has been shown to affect EEG asymmetry during sleep, ${ }^{16}$ and Casagrande and Bertini demonstrated a re-patterning of the laterality across wake-sleep state using the behavioral method. ${ }^{17}$

These cerebral asymmetries assign a greater dominance to one specific hemisphere-especially to the left side-according to motor and language functions. The left hemisphere is responsible for language functions, such as sentence construction, word fluency, while the right hemisphere is responsible 
for visuo-spatial attention. ${ }^{18-20}$

The purpose of this study is to investigate how electrophysiological appearance of cerebral asymmetry changes during the sleep onset period relative to the arousal state.

\section{METHODS}

\section{Subjects}

Sixty-one subjects with normal health status were recruited at a university hospital in Korea; there were thirty-four males with an average age of $26.6 \pm 2.4$ years, with a range of $23-34$ years, and twenty-seven females with an average age of $28.0 \pm$ 3.5 years, with a range of 23-35 years. All subjects were normal, healthy, and right-handed with normal EEG findings. Subjects were excluded from the study if they had any a history or current state of neurological disorders, psychiatric illnesses, cardiovascular diseases, pulmonary disorders, substance abuse, obstructive sleep apnea syndrome, and primary or secondary sleep disorders. Subjects with a history of alcohol consumption within 24 hours before the test were also excluded. The unreported depressive or anxiety symptoms of subjects were evaluated with Beck Depression Inventory (BDI) and Beck Anxiety Inventory (BAI), ${ }^{21,22}$ and subjects with BDI scores of greater than 13 or BAI scores of greater than 15 were excluded. We also excluded subjects who met the criteria for obesity classes I, II, and III, as defined by the World Health Organization, with a body mass index of more than $29 \mathrm{~kg} / \mathrm{m}^{2}$.

Subjects were instructed to perform sleep hygiene for a week prior to the EEG. Written consent was obtained from each participant. The study protocol was approved by the local Institutional Review Board (KUH1050056).

\section{Electroencephalography}

EEG measurements were taken between 10 am and 11 am by Neuroscan Version 4.3.3 (Compumedics, Abbotsford, Victoria, Australia). The international $10 \pm 20$ system with 32 channels were used, and the reference leads were placed in the ears on A1 and A2 sites. The laboratory was electrically shielded, protected against noise, and equipped with temperature and humidity control systems.

To eliminate the artifacts that possibly arise from the movements of the eyeball or eyelid, we put horizontal electro-oculography channels at 1-cm away from the outer portion of each eye and vertical electro-oculography channels above and below the left eye, and we also used a correction software embedded in Neuroscan. The EEG acquisition parameters were a sampling rate of $500 \mathrm{~Hz} / \mathrm{channel,} \mathrm{a} \mathrm{high-pass} \mathrm{filter} \mathrm{of} 0.05$ $\mathrm{Hz}$, a low-pass filter of $60 \mathrm{~Hz}$, and a notch filter of $60 \mathrm{~Hz}$.

The 30-second EEG measurement was performed on subjects with semi-supine positions and both eyes were closed.
Arousal (the wakeful state) and sleep state (the late stage 1 sleep) was distinguished by the distribution of the alpha and theta waves in $\mathrm{O} 1$ and $\mathrm{O} 2$ electrodes. The wakeful state was determined as the first 30-second epoch exhibiting a strong alpha pattern for the whole period. The late stage 1 sleep was defined as the final 30 seconds of stage 1 sleep.

\section{Statistical analysis}

Brain waves for the analysis were delta $(1-3.5 \mathrm{~Hz})$, theta (4$7.5 \mathrm{~Hz})$, alpha-1 (8-10 Hz), alpha-2 (10-12 Hz), beta-1 (12.5$18 \mathrm{~Hz})$, beta-2 $(18.5-21 \mathrm{~Hz})$, and beta-3 $(21.5-30 \mathrm{~Hz})$ bands. The power spectrum was calculated for 30 seconds of the time series data, and the asymmetry index was defined as $\log 10$ (the power of the left electrode)-log10 (the power of the right electrode). Statistical comparisons of the asymmetry index were performed between the two states for twelve electrode pairs (Fp1-Fp2, F3-F4, F7-F8, FC3-FC4, FT7-FT8, C3-4, T7-8, CP3-4, TP7-TP8, P3-P4, P7-P8, and O1-O2), and a statistical significance was set at $\mathrm{p}<0.01$.

\section{RESULTS}

\section{Left-right power difference (Table 1)}

In the wakeful state, the left hemisphere showed a greater power for alpha-2 at the FT7 and C3 electrodes and for beta2 at the CP3 electrode. Conversely, the right hemisphere showed predominance for alpha-1 at the FP2, F4, F8, P4, P8, and O2 electrodes, for alpha- 2 at the $\mathrm{O} 2$ electrode, and for beta- 2 at the F8 electrode.

In the late stage 1 sleep, the right hemisphere showed predominance for all EEG frequencies (delta at F4, theta at FP2, F4, F8, FT8, alpha-1 at FP2, F4, F8, O2, alpha-2 at FP2, F4, beta-1 at FP2, F4, beta-2 at FP2, F4, F8, beta-3 at FP2, F4, F8, FC4, FT8, and C4). No left hemispheric electrodes showed

Table 1. The electrodes that showed greater power than the contralateral ones

\begin{tabular}{|c|c|c|c|c|}
\hline & \multicolumn{2}{|c|}{ Wakefulness } & \multicolumn{2}{|r|}{ Stage 1 sleep } \\
\hline & Left & Right & Left & Right \\
\hline Delta & & & & $\mathrm{F} 4$ \\
\hline Theta & & & & FP2, F4, F8, FT8 \\
\hline Alpha-1 & & $\begin{array}{r}\mathrm{FP} 2, \mathrm{~F} 4, \mathrm{~F} 8 \\
\mathrm{P} 4, \mathrm{P} 8, \mathrm{O} 2\end{array}$ & & $\mathrm{FP} 2, \mathrm{~F} 4, \mathrm{~F} 8, \mathrm{O} 2$ \\
\hline Alpha-2 & FT7, C3 & $\mathrm{O} 2$ & & FP2, F4 \\
\hline Beta-1 & & & & FP2, F4 \\
\hline Beta-2 & $\mathrm{CP} 3$ & F8 & & $\mathrm{FP} 2, \mathrm{~F} 4, \mathrm{~F} 8$ \\
\hline Beta-3 & & & & $\begin{array}{l}\mathrm{FP} 2, \mathrm{~F} 4, \mathrm{~F} 8, \mathrm{FC} 4, \\
\text { FT8, C4 }\end{array}$ \\
\hline
\end{tabular}

Statics by paired t-test, $\mathrm{p}<0.01$, comparing the left and right leads in a state 


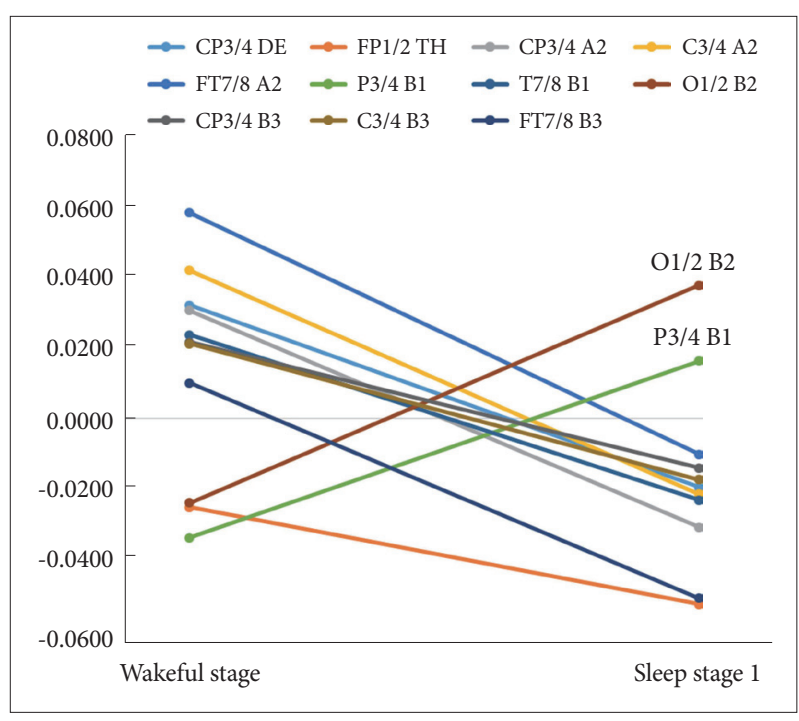

Figure 1. The change of asymmetric indices in the wakeful state and late-sleep stage 1 . The indices decreased in the late-sleep stage 1 compare to the wakeful state except the indices of beta-2 wave in 01/2 lead and beta-1 wave in P3/4 lead (statistics by paired t-test, $p<0.01$ ).

greater power than the right ones.

\section{Left-right and sleep state interaction (Figure 1)}

As consciousness changes from the wakeful state to the late stage 1 sleep, the asymmetry index of beta- 1 at the $\mathrm{P} 3 / 4$ electrode and of beta- 2 at the $\mathrm{O} 1 / 2$ electrode increased, but, were reduced for delta at the $\mathrm{CP} 3 / 4$ electrode, for theta at the FP1/2 electrode, for alpha- 2 at the FT7/8, C3/4, and CP3/4 electrodes, for beta- 1 at the $\mathrm{T} 7 / 8$ electrode, and for beta- 3 at the $\mathrm{CP} 3 / 4$ electrode.

\section{DISCUSSION}

Functional asymmetry of the human brain is prominent in handedness and language functions, ${ }^{23,24}$ and these functions tend to exhibit the dominance of the left hemisphere. However, the asymmetry of the brain is derived not only from those two functions. Cerebral asymmetry is a phenomenon not only in humans but also in other primates. In addition to the handedness and language function, there is also asymmetry in discriminating faces and recognizing sounds other than language. ${ }^{25-27}$ This asymmetry has been reported to differ between men and women. ${ }^{28-30}$ It has been found that the surface area of the corpus callosum compared to the brain volume is gradually smaller as it gets closer to human in primates, which is an important fact about the cause of brain asymmetry. ${ }^{31}$

In other words, as the amount of information exchanged between the right and left halves increases, the amount of brain volume must increase. Due to the anatomical limitations, the amount of information exchanged in the right and left halves cannot be increased without increasing the total brain capacity. Therefore minimizing the amount of information exchanged on the left and right is advantageous in allocating brain capacity to increase local brain function. This is why not only the functional differences in the right and left hemispheres but also the difference between the anterior and posterior parts of the brain must exist.

These asymmetries of the brain are, of course, reflected in functional brain imagings or various neuropsychological tests. Neuropsysiological indicators such as brain waves can, of course, reflect those. Most investigations about EEG asymmetry have studied frontal alpha rhythms. The meaning of alpha rhythm is still controversial, however the hypothesis holding that increased alpha power means the inactivity of the local cortical area was widely supported by various findings, such as increased alpha powers correlated with a decreased BOLD signal in brain areas, ${ }^{32}$ posterior alpha increases on eyes closed condition ${ }^{33}$ and motor cortical alpha increases with limbs at rest. ${ }^{34}$ Functionally, frontal alpha asymmetry have been suggested to be involved in emotional regulation or flexibility in response to psychological stress regardless of state or trait dependency. ${ }^{11,12}$ In that cases, left hemispheric dominance usually meant positive affective functioning.

On the transition from awakening to sleep, all parts of the brain do not fall asleep at the same time. There are some reports that the sleep proceeds to the posterior from the anterior of the brain, ${ }^{35,36}$ and other report that sleep onset process have different patterns according to the waveform of brain waves and cortical areas. ${ }^{37}$ Several reports suggested that the laterality of human brain can be changed or reversed during sleep. By behavioral method using finger tapping test, Casagrande and Bertini found the right side had short inter-tapping interval (ISI) in wakeful condition whereas the left hand showed shorter ISI during NREM and REM sleep. ${ }^{17}$ Left-right EEG asymmetry of the beta/delta ratio was reported to gradually reduced after sleep onset. ${ }^{38}$ Some studies found the right hemispheric power becoming predominated except beta, ${ }^{39-41}$ and two of these studies also reported a decrease in interhemispheric coherence during SOP. ${ }^{40,41}$

In this study, frontotemporal (FT7) and centroparietal (C3, CP3) electrodes had left side predominance in wakeful state, whereas right frontal, parietal and occipital electrodes became superior to the left side in sleep state. As for changes of the leftright asymmetry index, posteromedial beta oscillation showed increasing pattern, but most frontal, frontotemporal and central electrodes became more right predominance patterns during the sleep onset period. In summary, no left electrode was superior to the right in sleep state, and left-right asymmetry indices from five out of twelve electrode pairs decreased in five out of seven frequency bands. 
The reason why the laterality is different at the time of awakening and sleeping is not known precisely, but several hypotheses are known. The first is the so-called "Homeostatic hypothesis." This hypothesis is based on the restorative function of sleep, which is regarded as one of the most important functions of sleep. Because the dominant hemisphere has much more information and stress to be processed when being awake than the non-dominant hemisphere has, it should take more rest during sleep and this seems to make non-dominant hemispheres look relatively more active. ${ }^{17,42}$

The second hypothesis focuses on the intrinsic function of the non-dominant hemisphere itself and is also called the "Arousal hypothesis." It is known that the right hemisphere more contributes to the attention or sustained vigilance than the left hemisphere. ${ }^{17,43}$ In the sleep, the right hemisphere can be relatively dominant because the vigilance needs to be maintained somewhat relative to other functions. ${ }^{17}$

In this study, asymmetry index generally changed toward the right hemisphere dominant pattern during SOP. However, beta waves at the posterior medial electrodes showed different directions toward the left hemisphere. Despite the various limitations such as any possibility of the different SOP pattern between man and woman, though we can hardly find articles about it, and the possibility of hidden artifacts from relatively long recording time, the meaning of this study attributes to revealing the different asymmetry changing pattern of posterior medial beta waves from other parts of the brain during SOP with relatively many electrode pairs for all the usual bandwidth of EEG. However, further studies on the meaning and function of posterior beta waves are required to completely understand the reversal phenomenon of asymmetry.

\section{Acknowledgments}

This work was supported by the intramural research grant of Chungbuk National University in 2015.

\section{REFERENCES}

1. Teszner D, Tzavaras A, Gruner J, Hécaen H. Right-left asymmetry of the planum temporale; apropos of the anatomical study of 100 brains. Rev Neurol (Paris) 1972;126:444-449.

2. Rubens AB, Mahowald MW, Hutton JT. Asymmetry of lateral (Sylvian) fissures in man. Neurology 1976;26:620-624.

3. Galaburda AM, Corsiglia J, Rosen GD, Sherman GF. Planum temporale asymmetry: reappraisal since Geschwind and Levitsky. Neuropsychologia 1987;25:853-868.

4. Eidelberg D, Galaburda AM. Inferior parietal lobule. Divergent architectonic asymmetries in the human brain. Arch Neurol 1984;41:843852 .

5. Galaburda AM. Asymmetries of cerebral neuroanatomy. Ciba Found Symp 1991;162:219-226; discussion 226-233.

6. Benca RM, Obermeyer WH, Larson CL, Yun B, Dolski I, Kleist K, et al. EEG alpha power and alpha power asymmetry in sleep and wakefulness. Psychophysiology 1999;36:430-436.

7. Swanson N, Eichele T, Pearlson G, Kiehl K, Yu Q, Calhoun VD. Lateral differences in the default mode network in healthy controls and schizophrenia patients. Hum Brain Mapp 2011;32:654-664.

8. Bolduc C, Daoust AM, Limoges E, Braun CMJ, Godbout R. Hemispheric lateralization of the EEG during wakefulness and REM sleep in young healthy adults. Brain Cogn 2003;53:193-196.

9. Hagemann D, Hewig J, Seifert J, Naumann E, Bartussek D. The latent state-trait structure of resting EEG asymmetry: replication and extension. Psychophysiology 2005;42:740-752.

10. Jackson DC, Mueller CJ, Dolski I, Dalton KM, Nitschke JB, Urry HL, et al. Now you feel it, now you don't: frontal brain electrical asymmetry and individual differences in emotion regulation. Psychol Sci 2003;14: 612-617.

11. Papousek I, Reiser EM, Weber B, Freudenthaler HH, Schulter G. Frontal brain asymmetry and affective flexibility in an emotional contagion paradigm. Psychophysiology 2012;49:489-498.

12. Lewis RS, Weekes NY, Wang TH. The effect of a naturalistic stressor on frontal EEG asymmetry, stress, and health. Biol Psychol 2007;75:239247.

13. Kalin NH, Larson C, Shelton SE, Davidson RJ. Asymmetric frontal brain activity, cortisol, and behavior associated with fearful temperament in rhesus monkeys. Behav Neurosci 1998;112:286-292.

14. Tops M, Wijers AA, van Staveren ASJ, Bruin KJ, Den Boer JA, Meijman TF, et al. Acute cortisol administration modulates EEG alpha asymmetry in volunteers: relevance to depression. Biol Psychol 2005;69:181-193.

15. Tops M, van Peer JM, Wester AE, Wijers AA, Korf J. State-dependent regulation of cortical activity by cortisol: an EEG study. Neurosci Lett 2006;404:39-43.

16. Cajochen C, Biase RD, Imai M. Interhemispheric EEG asymmetries during unilateral bright-light exposure and subsequent sleep in humans. Am J Physiol Regul Integr Comp Physiol 2008;294:R1053-R1060.

17. Casagrande M, Bertini M. Night-time right hemisphere superiority and daytime left hemisphere superiority: a repatterning of laterality across wake-sleep-wake states. Biol Psychol 2008;77:337-342.

18. Laeng B, Shah J, Kosslyn S. Identifying objects in conventional and contorted poses: contributions of hemisphere-specific mechanisms. Cognition 1999;70:53-85.

19. Schmidt P, Krings T, Willmes K, Roessler F, Reul J, Thron A. Determination of cognitive hemispheric lateralization by "functional" transcranian Doppler cross-validated by functional MRI. Stroke 1999;30:939-945.

20. Burbaud P, Camus O, Guehl D, Bioulac B, Caille J, Allard M. Influence of cognitive strategies on the pattern of cortical activation during mental subtraction. A functional imaging study in human subjects. Neurosci Lett 2000;287:76-80.

21. Beck AT, Epstein N, Brown G, Steer RA. An inventory for measuring clinical anxiety: psychometric properties. J Consult Clin Psychol 1988; 56:893-897.

22. Beck AT, Ward CH, Mendelson M, Mock J, Erbaugh J. An inventory for measuring depression. Arch Gen Psychiatry 1961;4:561-471.

23. Raymond $M$, Pontier D. Is there geographical variation in human handedness? Laterality 2004;9:35-51.

24. Knecht S, Dräger B, Deppe M, Bobe L, Lohmann H, Flöel A, et al. Handedness and hemispheric language dominance in healthy humans. Brain 2000;123:2512-2518.

25. Hopkins WD. Comparing human and nonhuman primate handedness: challenges and a modest proposal for concensus. Dev Psychobiol 2013; 55:621-636.

26. Hamilton CR, Vermeire BA. Complementary hemispheric specialization in monkeys. Science 1988;242:1691-1694.

27. Pohl P. Central auditory processing: V. Ear advantage for acoustic stimuli in baboons. Brain Lang 1983;20:44-53.

28. De Lacoste-Utamsing C, Holloway RL. Sexual dismorphism in the human corpus callosum. Science 1982;216:1431-1432.

29. Driesen NR, Raz N. The influence of sex, age and handedness on corpus callosum morphology: a metal-analysis. Psychobiology 1995;23:240-247.

30. Holloway RL, de Lacoste MC. Sexual dimorphism in the human corpus 
callosum: an extension and replication study. Human Neurobiol 1986; 5:87-91.

31. Hopkins WD, Misiura M, Pope SM, Latash EM. Behavioral and brain asymmetries in primates: a preliminary evaluation of two evolutionary hypotheses. Ann N Y Acad Sci 2015;1359:65-83.

32. Goldman RI, Stern JM, Engel J Jr, Cohen MS. Simultaneous EEG and fMRI of the alpha rhythm. Neuroreport 2002;13:2487-2492.

33. Treder MS, Bahramisharif A, Schmidt NM, van Gerven MA, Blankertz B. Brain-computer interfacing using modulations of alpha activity induced bycovert shifts of attention. J Neuroeng Rehabil 2011;8:24.

34. Sterman MB, Egner T. Foundation and practice of neurofeedback for thetreatment of epilepsy. Appl Psychophysiol Biofeedback 2006;31:2135 .

35. Wright KP, Badia P, Wauquier A. Topographical and temporal patterns of brain activity during the transition from wakefulness to sleep. Sleep 1995; 18:880-889.

36. Tanaka H, Hayashi M, Hori T. Topographical characteristics and principal component structure of the hypnagogic EGG. Sleep 1997;20:523534.

37. Park DH, Ha JH, Ryu SH, Yu JH, Shin CJ. Three-dimensional electro- encephalographic changes on Low-Resolution Brain Electromagnetic Tomography (LORETA) during the sleep onset period. Clin EEG Neurosci 2015;46:340-346

38. Merica H, Gaillard JM. The EEG of the sleep onset period in insomnia: a discriminant analysis. Physiol Behav 1992;52:199-204.

39. Boldyreva GN, Zhavoronkova LA. Interhemispheric asymmetry of EEG coherence as a reflection of different functional states of the human brain. Biomed Sci 1991;2:266-270.

40. Zhavoronkova LA, Trofimova EV. Coherence dynamics of EEG and motor reactions while falling asleep in right-handed and left handed people I. Analysis of interhemispheric correlations. Fiziol Cheloveka 1997;23:18-26.

41. Zhavoronkova LA, Trofimova EV. Dynamics of EEG coherence in righthanders and left-handers when falling asleep: II. An analysis of interhemispheric relations. Human Physiol 1998;24:32-39.

42. Violani C, Testa P, Casagrande M. Actigraphic motor asymmetries during sleep. Sleep 1998;21:472-476.

43. Jalili M. Hemispheric asymmetry of electroencephalography-based functional brain networks. Neuroreport 2014;25:1266-1271. 